\title{
Number of Units
}

National Cancer Institute

\section{Source}

National Cancer Institute. Number of Units. NCI Thesaurus. Code C158697.

The number of units. 